\title{
DECISÕES DE CONSUMO E INVESTIMENTO: COMPARAÇÃO DAS TAXAS DE JUROS DE CRÉDITO PARA ELETRODOMÉSTICOS ENTRE DOIS SHOPPINGS DE NATAL/RN
}

\author{
M. P. de Lima Jr¹, S. S. de L. da Costa², C. B. da C. Mota Filha², K. T. X. de S. Patrício², \\ R. da S. Moura ${ }^{2}$ \\ ${ }^{1}$ Instituto Federal do Rio Grande do Norte - Campus Natal Central \\ melquiades.pereira@ifrn.edu.br, \\ ${ }^{2}$ Instituto Federal do Rio Grande do Norte - Campus Natal Zona Norte
}

Artigo submetido em dezembro/2009 e aceito em abril/2011

\section{RESUMO}

Este trabalho teve como objetivo verificar por meio de uma pesquisa de mercado a comparação das taxas de juros de eletrodomésticos entre as lojas pertencentes aos centros comerciais Shopping Midway e Norte Shopping. A pesquisa foi realizada analisando as taxas de crediário de eletrodomésticos nas lojas dos dois centros comerciais a fim de estabelecer uma comparação entre as médias das taxas de juros em ambos os locais, testando a hipótese de que as médias das taxas são estatisticamente iguais. Assim, para a execução da pesquisa foi estratificada uma amostra de taxas de juros, dividida em dois grupos e comparadas, utilizando o teste não-paramétrico denominado Mann-Whitney. Concluímos que no comércio local, onde foi delimitado o estudo, as lojas mantêm um padrão, e o resultado do teste demonstra com 95\% de confiança, que na média as taxas de juros são estatisticamente iguais. Desta maneira, de acordo com a pesquisa as taxas de juros nos dois locais não diferem na média, isso comprova a viabilidade de que não é necessário que o consumidor se desloque de uma região a outra em busca de condições de compra mais acessíveis, ao se tratar de taxa de juros.

PALAVRAS-CHAVE: Juros, Consumo, Crédito

\section{CONSUMPTION AND INVESTMENT DECISIONS: A COMPARISON OF INTEREST RATES ON CREDIT FOR APPLIANCES BETWEEN TWO MALLS FOR NATAL / RN}

\begin{abstract}
This study aimed to verify through a market survey to compare the interest rates of home appliances stores between Midway and North Shopping Mall. The survey was conducted by analyzing the rates of payment plans appliances in stores in two malls in order to establish a comparison between the average interest rate of home appliances in both locations, testing the hypothesis that the mean rates are statistically equal. Thus, for the implementation of the survey was a stratified sample of interest rates, divided into two groups and compared the averages are statistically significant using the nonparametric test Mann-Whitney. We conclude that in the globalized world shops keep a standard, and the test result shows, with 95\% confidence that the average interest rates are statistically equal. Thus, according to research interest rates in dduas regions do not differ on average, that proves not feasible consumer moves from one region to another in search of more affordable purchase terms, in the case of interest rate.
\end{abstract}

KEY-WORDS: Interest Rate, Consumption and Loan 


\section{DECISÕES DE CONSUMO E INVESTIMENTO: COMPARAÇÃO DAS TAXAS DE JUROS DE CRÉDITO PARA ELETRODOMÉSTICOS ENTRE DOIS SHOPPINGS DE NATAL/RN}

\section{INTRODUÇÃO}

A história dos juros, aplicados nas atividades de vendas, teve início, mais precisamente, no final da Idade Média, com o surgimento da burguesia. Ao longo do tempo a busca pelo mercado consumidor foi aumentando na mesma proporção em que o sistema econômico vinha ganhando espaço no mundo.

A constante busca por mercados consumidores fez com que as potencias européias cruzassem as fronteiras continentais e estabelecessem suas multinacionais por todo o mundo. Antes, determinados produtos eram monopolizados por determinada empresa, enquanto que outras não poderiam vender o mesmo produto a fim de evitar a concorrência no mercado.

Com o advento da globalização, essa realidade mudou. Várias empresas passaram a vender os mesmos produtos em todo o mundo, inclusive via internet. Isso gerou certo "poder" por parte do consumidor, que agora passou a ter a opção de escolher o mesmo produto em vários mercados, procurando sempre o local mais barato. Fato esse que obrigou os mercados a entrarem em disputa por consumidores, oferecendo promoções e mais ofertas, a fim de vender mais e arrecadar lucros.

Essa grande disputa por clientes ocasionou na criação de uma nova forma de pagamento: o pagamento a prazo. Nessa forma de pagamento o cliente pode dividir o pagamento em várias vezes mensais até completar o preço "real" do produto. Mas, como o mercado não perde a oportunidade de sair no lucro, até a forma de pagamento a prazo chega a gerar lucros para a empresa que utiliza tal forma de pagamento.

Então entram os juros. A quantificação dos juros difere entre os mercados, entre produtos, segmentos e outros. Alguns exploram juros simples, outros juros compostos (regimes de cálculo dos juros diferenciados), o primeiro sendo utilizado para pequenos cálculos de juros ao consumidor final e o segundo é o caso mais comum em empréstimos bancários e concessão de crediário. No final de tudo o mercado que vende o produto lucra com essa forma de pagamento (ASSAF NETO, 2008).

Uma das cidades brasileiras onde o progresso está gerando esse tipo de concorrência no mercado consumidor é Natal-RN. A capital potiguar vem crescendo muito nesses últimos anos. Antes havia apenas um grande shopping (Natal Shopping Center). Mas essa realidade mudou: o Shopping Midway surgiu com novas lojas e mais opções de consumo. Recentemente, a zona norte de Natal recebeu o Natal Norte Shopping, intensificando ainda mais a grande disputa dos mercados por clientes e desses últimos por melhores ofertas. A partir daí o presente trabalho realizou uma pesquisa de preços de alguns eletrodomésticos para se fazer uma comparação entre as taxas de juros de crediário cobradas nos seguintes centros comerciais: as lojas do Natal Norte Shopping e as lojas do Shopping Midway. Foram tomados como exemplo produtos de eletrodomésticos (CASCUDO, 1999; TAVARES; ARAUJO, 2008; ASSUNCAO; ARAUJO, 2008).

A investigação e apresentação dos conhecimentos do conteúdo teórico sobre juros, aplicado as etapas de desenvolvimento do trabalho, se dão por meio da comparação: se na média, as taxas 
de juros de crediário em produtos de eletrodomésticos são estatisticamente iguais nos dois shoppings. A hipótese surgiu de acordo com diálogo com os lojistas, em que as taxas de juros cobradas em um dos centros comerciais, da região norte, não diferem das taxas cobradas pelos lojistas na região sul. Isso comprovaria a inviabilidade do consumidor que reside na região da zona norte vá até centros comerciais mais distantes, em outras regiões, para buscar condições de compra mais accessíveis, o que ocorre constantemente.

\section{COMPORTAMENTO DO CONSUMIDOR}

De acordo com Mowen e Minor (2003) o comportamento do consumidor é definido como o estudo das unidades compradoras e dos processos de troca envolvidos na aquisição, no consumo e na disposição de mercadorias, serviços, experiências e idéias. Para Engel (2000) comportamento do consumidor é o somatório das atividades diretamente envolvidas em obter, consumir e dispor de produtos e serviços, incluindo os processos decisórios que antecedem e sucedem estas ações. Assim, para entender melhor o comportamento do consumidor faz-se necessário conhecer o processo de compra e os fatores que influenciam na decisão.

Segundo Hisrich (2000), o processo de compra pode ser dividido em cinco estágios, a saber: reconhecimento da necessidade; busca de informações; avaliação das alternativas; decisão de compra e comportamento pós-compra. De acordo com Churchill (2000), o processo de tomada de decisão começa com o consumidor reconhecendo um problema a ser resolvido ou necessidade a ser satisfeita. O segundo estágio pode ser definido segundo Engel (2000) conceitua como a ativação motivada do conhecimento na memória ou da aquisição de informação do ambiente, cuja natureza pode ser interna, que envolve a recuperação de conhecimento da memória e a busca externa, que consiste em coletar informações do mercado (ENGEL, 2000). O terceiro estágio é definido por Churchill (2000), como a fase de avaliação da melhor alternativa a ser escolhida com base nos dados e informações coletadas no estágio anterior. O quarto estágio é o ato da compra e o último é o resultado da compra que pode gerar satisfação e fidelização do consumidor ou ocasionar insatisfação (MOWEN \& MINOR, 2003).

No âmbito dos fatores influenciadores da decisão Kotler (1998), descreve vários fatores causadores de influência conforme ilustrado na figura 1 .

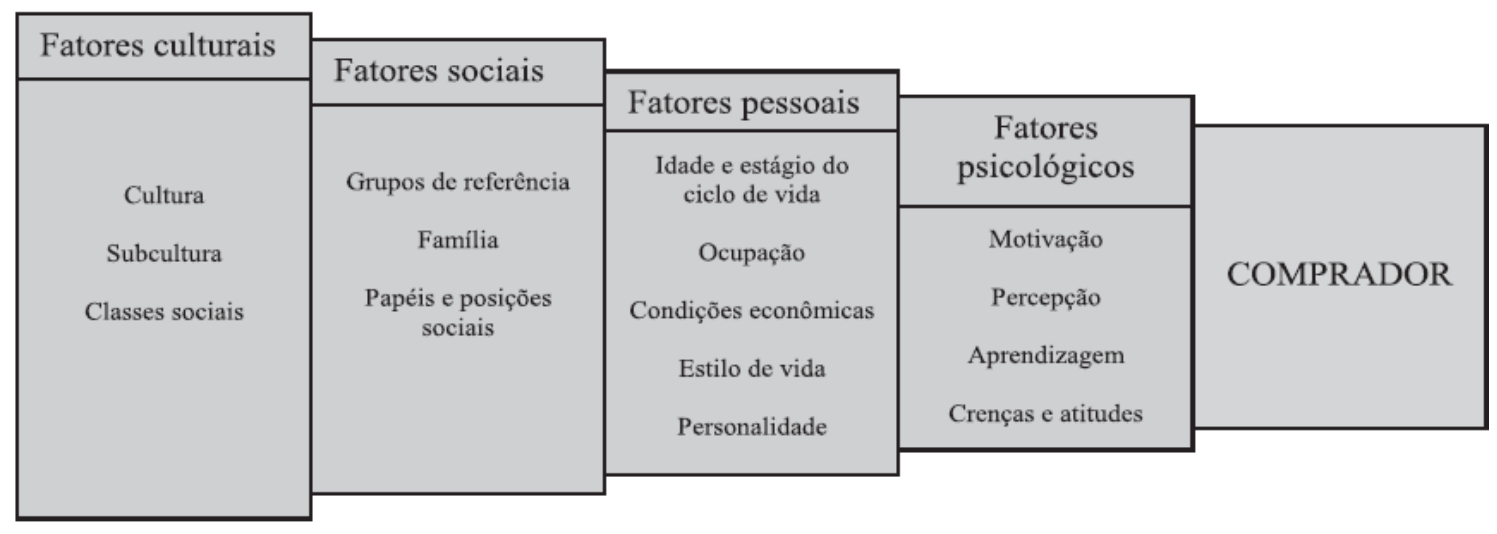

Figura 1 - Fatores de influência no processo de decisão de compra 


\section{JUROS E CONSUMO}

O cálculo financeiro estuda as relações que envolvem unidades monetárias em diferentes pontos no tempo. É por meio do cálculo financeiro e condições prevalentes no mercado que determina o valor da prestação. Assim, segundo Faro (2006) o cálculo financeiro determina a quantia que deve ser paga como prestação.

Faro (2006) conceitua juro como a remuneração, a qualquer título, atribuída ao fator capital. A determinação do valor do juro que é cobrado em qualquer transação financeira é efetuada mediante a consideração de um coeficiente "taxa de juro". As taxas de juros costumam ser apresentadas sob uma das duas seguintes formas: forma unitária e percentual.

Os juros induzem o adiantamento do consumo, permitindo a formação de poupanças e novos investimentos na economia. Os juros eficientes remuneram: risco da operação, incerteza, com o futuro; perda do poder pela inflação; e o ganho na aplicação. Os juros podem ser simples ou compostos. Os juros simples é aquele pago unicamente sobre o capital inicial, também chamado principal. O regime de juro compostos segue o princípio natural de que os juros formados a cada período rendem juros nos períodos seguintes, ou seja, os juros devidos ao capital que tínhamos no início desse período são incorporados àquele capital (ASSAF NETO, 2008).

\section{SÉRIES DE PAGAMENTOS E TAXA DE JUROS}

Um fluxo de caixa representa uma série de pagamentos ou de recebimentos que se estima ocorrer em determinado intervalo de tempo, as parcelas de pagamento. (ASSAF, 2008). Assim, defrontamos com operações financeiras que se representam por um fluxo de caixa, como diferentes tipos de financiamentos costumam envolver uma seqüência de desembolsos periódicos de caixa.

Segundo Faro (2006), as seqüências ditas periódicas, são aquelas em que o intervalo de tempo entre pagamentos consecutivos é constante, um exemplo prático é a concessão de crédito para a compra de um bem como eletrodoméstico em várias parcelas, os intervalos de tempo são sempre mensais. $\mathrm{O}$ número de pagamento é finito nas seqüências temporais, ou seja, as parcelas possuem um determinado limite de tempo.

As sequiências classificam também, em relação à data tomada como origem, em: postecipadas quando o primeiro pagamento ocorre no fim do primeiro período; e antecipadas quando o primeiro pagamento tem data de vencimento no inicio do primeiro período, como mostram as Figuras 2 e 3.

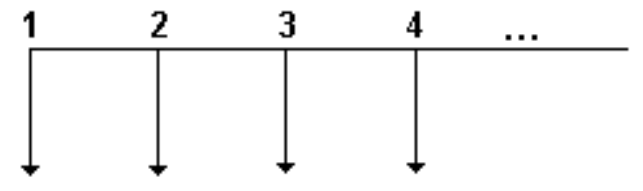

Figura 2: Pagamentos no início dos períodos: Fluxo ANTECIPADO

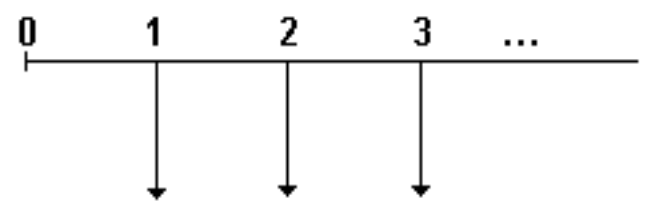

Figura 3: Pagamentos no final dos períodos: Fluxo POSTECIPADO 
Os pagamentos de caixa podem ser Constantes referindo-se a valores, isso se dá se os fluxos de caixa apresentados sejam iguais, ou Variáveis caso os fluxos de caixa não forem sempre iguais entre si. No entanto, se os valores de caixa apresentarem desigualdade, variáveis, o valor presente é calculado pela soma dos valores atualizados de cada um de seus termos. $\mathrm{O}$ valor presente de um fluxo de caixa uniforme para uma taxa periódica de juros é determinado pelo somatório dos valores presentes de cada um de seus valores, como é descrito na Equação 1.

\section{$\mathbf{P V}=\mathbf{P M T} \times \mathbf{F P V}(\mathbf{i}, \mathbf{n})$}

(1)

Onde:

$\mathrm{Pv}=$ Present Value ou Valor a Vista

PMT = Payment ou parcela a prazo

$\mathrm{i}=$ interest rate ou taxa de juros

$\mathrm{n}=$ prazo

FPV = Fator de Valor Presente que compõe i e n (ou coeficiente de financiamento)

Observe que FPV equipara-se à soma de uma progressão geométrica (PG) de $n$ termos, sendo o primeiro termo $\left(\mathrm{a}_{1}\right)$ e a razão $(\mathrm{q})$ igual a $(1+\mathrm{i})^{-1}$, e o n-ésimo termo $\left(\mathrm{a}_{n}\right)$ igual a $(1+\mathrm{i})^{-n}$.

Em suma, a base de comparação para se definir uma carência é o final do primeiro período. A periodicidade reflete os intervalos de tempo em que os fluxos de caixa ocorrem. Para isso a expressão de cálculo do coeficiente de financiamento é desenvolvida a partir da fórmula do valor presente padrão dos fluxos de caixa. Representa a Equação 2.

$\operatorname{FPV}(\mathbf{i}, \mathbf{n})=\frac{(1+\mathbf{i})^{\mathrm{n}}-1}{\operatorname{ix}(1+\mathrm{i})^{\mathrm{n}}} \quad$ ou $\quad \operatorname{FPV}(\mathbf{i}, \mathbf{n})=\frac{1-(1+\mathbf{i})^{-n}}{\mathbf{i}}$

Equação (2)

Conseqüentemente o cálculo da parcela de pagamento a partir do valor presente (valor a vista do bem) é descrito pela Equação 3.

$$
\text { PMT }=\text { PV } x \frac{1}{\operatorname{FPV}(\mathbf{i}, \mathbf{n})}
$$

Equação (3)

Como também a taxa de juros pode ser encontrada considerando o valor inicial (a vista do bem), o valor da parcela e a quantidade de parcelas na aquisição a prazo. O cálculo da taxa de juros em uma série uniforme de pagamentos postecipada ou mesmo antecipada não pode ser encontrado por meio de uma fórmula simples.

É um caso típico para se contar com o apoio de uma calculadora como a HP12c ou o apoio de um software, como uma planilha eletrônica, Microsoft Excel ${ }^{\circledR}$. Por mais que se tente chegar 
à solução pelo método algébrico, somente conseguiremos uma taxa estimada, que servirá de base para iniciarmos um processo de tentativa e erro.

Conforme foi exposto, o coeficiente de financiamento FPV embute os juros definidos para a operação. No entanto, outras despesas podem ainda ser consideradas no fator financeiro, tais como IOF, taxa de abertura de crédito etc.

\section{METODOLOGIA}

O trabalho pôde ser caracterizado como uma pesquisa quantitativa e mais especificamente de campo em torno das taxas de juros cobradas por operações de crédito na venda a prazo de eletrodomésticos, a pesquisa foi realizada mais especificamente em dois centros comerciais, o Natal Norte Shopping e o Shopping Midway (ROESCH, 1999).

A pesquisa foi caracterizada como quantitativa por envolver a utilização e comparação de dados métricos, como também como pesquisa de corte transversal por coletar os dados em um determinado momento do tempo. A distinção da variável taxa de juros foi realizada por meio de uma variável do tipo dicotômica (0 e 1), caracterizando as duas localidades.

Segundo Roesch (1999) para a execução da pesquisa foi estratificada uma amostra de taxas de juros, e esta foi dividida em dois grupos, um do Shopping Midway e outra do Norte Shopping ambos em Natal/RN. Estes dois grupos foram comparados em suas médias para se analisar se as diferenças são estatisticamente relevantes. O período de coleta compreendeu entre 26/09 e $11 / 10$ de 2009, este período estima-se que não houve nenhum viés em relação a alterações econômicas, foi certificado de que não houve liquidações, alterações de preços e nem alterações da taxa de juros pelo governo, de acordo com o site DEBIT (2009) o valor simples da taxa Selic do mês de agosto a outubro de 2009 permaneceu constante, o que demonstra a estabilidade nas taxas de juros do mercado nesse período.

Os dados foram levantados de forma empírica por meio de formulário elaborado previamente, sendo coletadas as seguintes informações por conveniência: preço a vista, valor da parcela e quantidade das parcelas de compra a prazo por meio de crediário. As informações foram tabuladas e tratadas de forma que permitiu encontrar qual a taxa de juros cobrada na venda do produto no crediário (BARBETTA, 2005).

\section{ANALISE DOS RESULTADOS}

A população foi de difícil estimação. Considerando a probabilidade de que cada produto em cada loja e em cada shopping tenha uma taxa de juros diferenciada, então o cálculo probabilístico da população foi baseado como segue nas Equações 4 e 5 (BARBETTA, 2005):

\section{População $=$ NS $x$ NTP $x$ NL $x$ NMP}

Equação (4)

População $=2 \times 7 \times 4 \times 20=1.120$

Equação (5) 
Em que:

NS $=N^{o}$ Shoppings (Norte Shopping e Shopping Midway)

$\mathrm{NTP}=\mathrm{N}^{\mathrm{o}}$ de Tipos de Produtos (Fogão, Refrigerador, Lavadoura, etc.)

$\mathrm{NL}=\mathrm{N}^{\mathrm{o}}$ de Lojas (4 Lojas em cada shopping que comercializam eletrodomésticos)

NMP $=\mathrm{N}^{\circ}$ Médio de Modelos por Tipo de Produtos (Refrigerador Consul 4505, Refrigerador Brastemp 1080, Fogão Consul Erva-Doce, etc.)

Assim, de acordo com Barbetta (2005) a amostra a ser pesquisada foi calculada da seguinte forma (foi considerado um erro amostral de $11 \%$ ):

$n=\frac{N \cdot\left(1 / E_{0}^{2}\right)}{N+\left(1 / E_{0}^{2}\right)}=77$

$\mathrm{n}=$ Amostra

$\mathrm{N}=$ População

$E_{0}^{2}=$ Erro Amostral ao Quadrado

Os dados então demonstraram as seguintes características com relação a sua estatística:

Tabela 1: Estatística Descritiva dos Dados

\begin{tabular}{|c|c|c|c|c|}
\hline Estatística Descritiva & N & Média & Desvio Padrão & Variância \\
\hline Taxa de Juros & 78 & 0,0267126 & 0,00997878 & 0,00009958 \\
\hline Grupo Zona Norte & 33 & 0,0260515 & 0,00146040 & 0,00000213 \\
\hline Grupo Zona Sul & 45 & 0,0271973 & 0,00164994 & 0,00000272 \\
\hline
\end{tabular}

A linha da Taxa de Juros da Tabela 1 compreende a estatística descritiva da amostra realizada, contendo 78 observações, destre estas 33 foram observações coletadas no Norte Shopping e 45 observações coletadas no Shopping Midway. Dentre as estatísticas elaboradas no quadro foi perceptível de que as taxas de juros possuem médias e variabilidade bem próximas, o que constataria préviamente de que não há uma diferença estatísticamente significante entre as médias. Para comprovação mais consistente foi realizada a análise dos dados por meio de testes apropriados.

Para a análise da comparação entre as médias foi previsto inicialmente a realização da ANOVA - Análise de Variância, que tem como objetivo comparar se a distância entre as médias de dois grupos é estatisticamente significante. Para isso a ANOVA possui dois pressupostos para a sua realização que são: normalidade dos dados e a igualdade entre as variâncias.

Para a análise da igualdade das variâncias utilizou-se o teste de Levene, conforme Tabela 2. 
Tabela 2: Estatística do Teste de Homogeneidade das Variâncias

\begin{tabular}{|r|r|r|r|}
\hline \multicolumn{4}{|c|}{ Teste de Homogeneidade das Variâncias } \\
\hline $\begin{array}{c}\text { Estatística } \\
\text { Levene }\end{array}$ & df1 & df2 & Sig. \\
\hline 1,523 & 1 & 76 & 0,221 \\
\hline
\end{tabular}

O teste então conforme Sig. $>0,05$ atestou que a igualdade entre as variâncias é estatisticamente significativa a $95 \%$ de confiança. Apresenta então que o primeiro pressuposto da ANOVA foi aceito.

Em seguida foi analisada a normalidade dos dados (taxas de juros), por meio do teste Kolmogorov-Smirnov, o que constatou, com $95 \%$ de confiança, que os dados não apresentaram distribuição normal (hipótese $\mathrm{H}_{0}$ de normalidade rejeitada Sig.<0,05). Conseqüentemente o segundo pressuposto da ANOVA não foi aceito (normalidade), o que faz com que seja utilizado um teste não-paramétrico. O resultado do K-S é demonstrado na Tabela 3, o teste também é reforçado pelo gráfico demonstrado na Figura 4 em função da distância das observações, pontos, em relação a linha traçada, como também pela Figura 5 que mostra a discrepância do histograma dos dados em relação a linha de densidade normal.

Tabela 3: Estatística do Teste de Normalidade

\begin{tabular}{|l|r|c|}
\hline \multicolumn{3}{|c|}{ Teste de Normalidade } \\
\hline \multicolumn{3}{|c|}{ Kolmogorov-Smirnov } \\
\hline Variável & Estatística & Sig. \\
\hline Taxa & 0,239 & 0,000 \\
\hline
\end{tabular}

Normal Q-Q Plot of Taxa

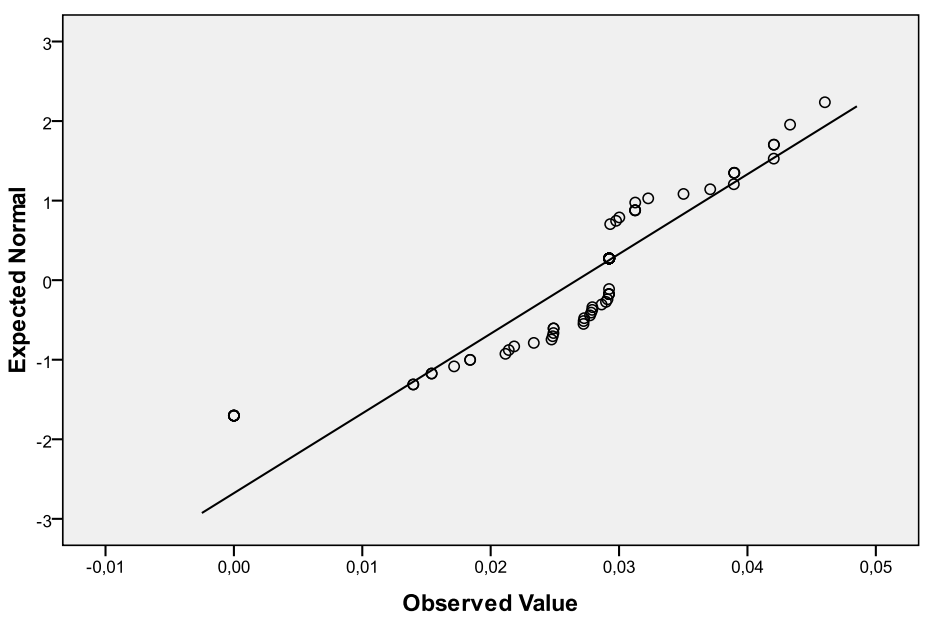

Figura 4: Gráfico de normalidade Q-Q 


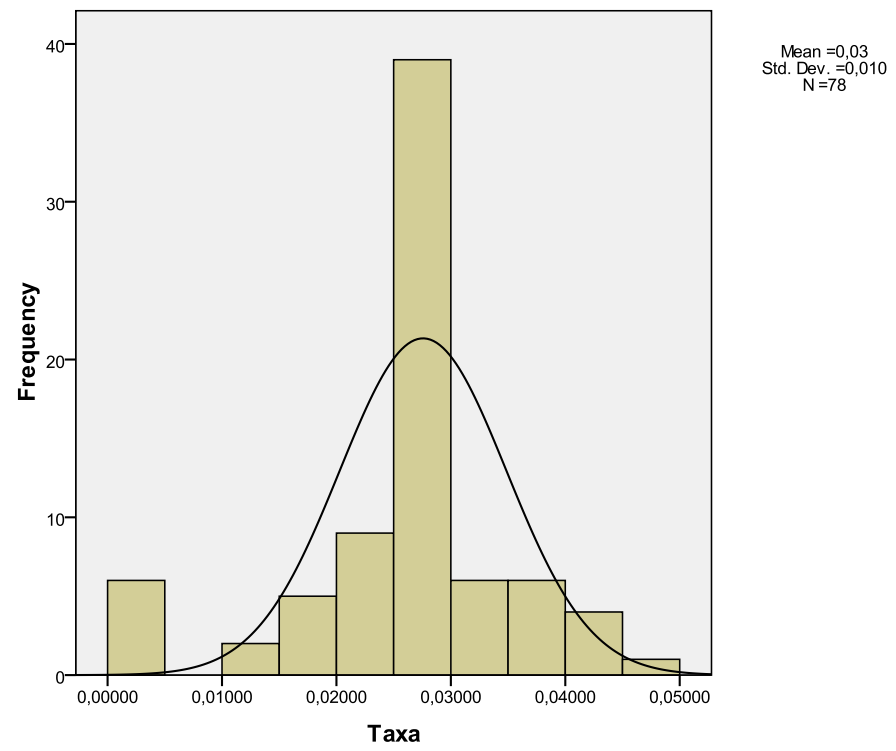

Figura 5: Gráfico do histograma com a linha da densidade normal

Então, como foi constatado que os dados não possuem uma distribuição normal, Morgan et al (2004) sugere a utilização de um teste não-paramétrico, que é um teste para dados com distribuição não normal. Assim o teste utilizado foi o Mann-Whitney que é um teste de comparação de médias utilizado quando o pressuposto de normalidade dos dados da ANOVA é quebrado (a comparação da igualdade entre as médias é realizada por meio da análise das variâncias entre os grupos), como também quando se pretende comparar média de grupos independentes, que foi este caso.

No caso do Teste Mann-Whitney seus pressupostos são: que a variável dependente seja contínua e que os grupos sejam independentes entre si. Estes então foram perfeitamente aceitos por meio das características dos dados coletados (MORGAN; et al, 2004). O resultado do teste é demonstrado pela Tabela 4.

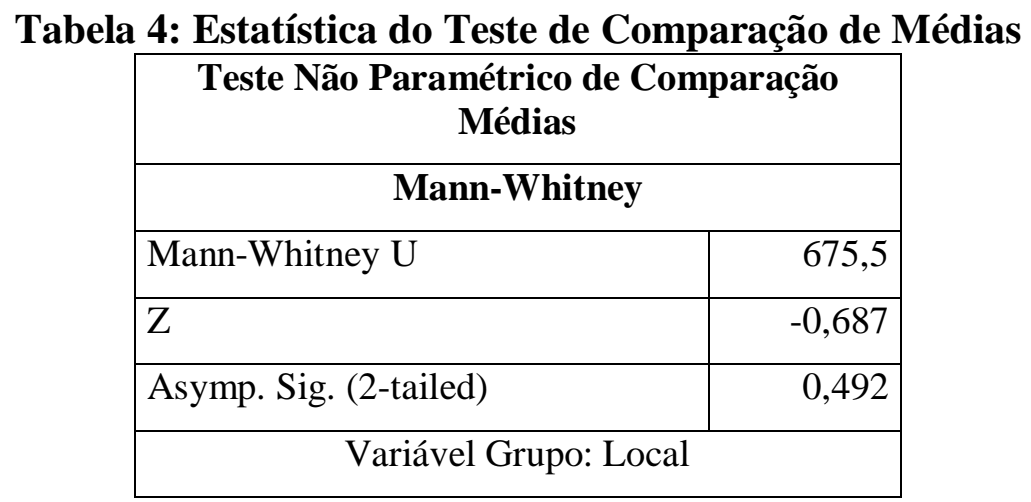

Então, as hipóteses a serem testadas foram

$\mathrm{H}_{0}$ : Os dados possuem média estatisticamente iguais

$\mathrm{H}_{1}$ : Os dados possuem média estatisticamente diferentes

O resultado do teste demonstra, com $95 \%$ de confiança, como o Sig.>0,05 a hipótese nula não é estatisticamente relevante para ser rejeitada, o que resulta que na média, a diferença entre os dois grupos não é estatisticamente significativa. Então as lojas situadas nos dois shoppings 
que vendem produtos de eletrodomésticos a prazo com crediário cobram, em média, taxas de juros aproximadamente iguais.

Levando em consideração como o estudo que foi realizado, podem-se constatar algumas limitações, como: a escolha por conveniência e a utilização de determinados tipos de eletrodomésticos. Um ponto relevante para esta pesquisa é que foi realizado um censo de todas as lojas de eletrodomésticos dos dois centros comerciais, como também dos tipos de produtos comercializados. Por se tratar de uma pesquisa de caráter empírica existe a interferência de variáveis de confundimento como diferentes modalidades de pagamento a prazo, como cartões de crédito, crediário e outros; como também de diferentes operadoras de cartão de crédito. Como se tratou de uma pesquisa não probabilística não se pode extrapolar os resultados para a população alvo do estudo, ou seja, possibilitou apenas inferir os resultados para as lojas e produtos participantes do estudo.

\section{CONSIDERAÇÕES FINAIS}

Após a chegada de mais lojas na cidade, o consumidor passou a ter mais opções de compras. Em decorrência disso verificou-se que as lojas foram forçadas a oferecer promoções e novas formas de pagamento a fim de ganhar mais clientes. Essas formas de conseguir clientes proporcionaram uma taxa de juros cobrada a partir de todo capital emprestado por certo período de tempo, com o objetivo de facilitar a aquisição do consumidor.

Apesar de haver certa competitividade entre as lojas do Midway Mall e do Natal Norte Shopping, é perceptível que a diferença das taxas de juros é muito pequena, quando comparadas. De uma maneira geral, pode-se considerar que, apesar da concorrência proposta pelo mercado, as lojas mantêm, entre si, certo padrão em relação ao preço de mercado dos produtos e suas condições de crédito. O deslocamento de uma região a outra não seria exatamente a melhor forma de se economizar, caso o cliente esteja visando isso, para a aquisição dos produtos de eletrodomésticos.

Concluí-se então que as duas localidades possuem taxas de juros de crediários realmente muito semelhantes entre si, quando comparadas em suas médias os grupos apresentam igualdade estatisticamente significante. $\mathrm{O}$ que faz com que não seja necessário o consumidor que reside na zona norte buscar pelo centro comercial mais distante na região sul. $\mathrm{O}$ consumidor dentre outras facilidades economiza tempo e recursos de locomoção que seriam necessários ao acesso às demais regiões da cidade, o que comprova o desenvolvimento urbano e econômico de localidades mais distantes em Natal.

\section{REFERÊNCIAS BIBLIOGRÁFICAS}

1. ASSAF NETO, A. Matemática Financeira e suas aplicações. 10 ed. São Paulo: Atlas, 2008.

2. ASSUNCAO, J.C.F.; ARAUJO, M.C.C. Pelo direito de ir e vir na cidade: mobilidade urbana e inclusão social em cidade praia - Natal/RN. Revista Holos, v. 1, p. 48-74, 2008.

3. BARBETTA, Pedro Alberto. Estatística Aplicada às Ciências Sociais. 5 ed. Florianópolis: Ed. da UFSC, 2005.

4. CASCUDO, L.C. História da cidade do Natal: RN Econômico. 1999. 
5. CHURCHILL, Gilbert A. Marketing: criando valor para o cliente. São Paulo: Saraiva, 2000.

6. DEBIT. Histórico de Índices Acumulados. São Paulo: DEBIT, 2009. Disponível em: <http://www.debit.com.br/consulta10.php>. Acesso em: 21 jan. 2009.

7. ENGEL, J. F.; BLACKWELL, R. E.; MINIARDI, P. W. Comportamento do consumidor. Rio de Janeiro: LTC Editora, 2000.

8. FARO, C. Fundamentos de Matemática Financeira: uma introdução ao cálculo financeiro e a analise de investimento de risco. São Paulo: Saraiva, 2006.

9. HISRICH, Robert D. Marketing. 2 ed. New York: Barron`s Business Library, 2000.

10. KOTLER, Philip. Administração de marketing: análise, planejamento, implementação e controle. 5. ed. São Paulo: Atlas, 1998.

11. MORGAN, G.A. et al. SPSS For Introductory Statistics: use and interpretation. 2 ed. London: Lawrence Erlbaum Associates, 2004.

12. MOWEN, John C.; MINOR, Michael S. Comportamento do consumidor. São Paulo: Prentice Hall, 2003.

13. ROESCH, S.M.A. Projetos de estágio e de pesquisa em administração. 2 ed. São Paulo: Atlas, 1999.

14. TAVARES, M.A.A.; ARAUJO, M.C.C. Os condomínios Green Village e Green Woods e as transformações do espaço urbano de Natal. Revista Holos, v. 1, p.75-92, 2008 . 